\title{
MASSAGEM NA SAÚDE LABORAL: INFLUÊNCIA NO COMPORTAMENTO MOTOR E ESTRESSE OCUPACIONAL
}

\author{
MASAJE EN LA SALUD LABORAL: INFLUENCIA \\ EN EL COMPORTAMIENTO MOTOR Y EL ESTRÉS \\ OCUPACIONAL
}

\section{MASSAGE ON LABOR HEALTH: INFLUENCE ON MOTOR BEHAVIOR AND OCCUPATIONAL STRESS}

\author{
GERALDO MAGELA DURÃES \\ Doutor pela Universidade Tras-os-Montes e Alto Douro (Portugal) \\ Professor Universidade Estadual de Montes Claros, Grupo de Pesquisa \\ em psicologia do sporte, exerc cio esa de ocupacional e m dia GIPESOM \\ (Montes Claros - Brasil) \\ gmdmoc@yahoo.com.br
}

\section{JEAN CLAUDE LAFETÁ}

Doutor pela Universidade Tras-os-Montes e Alto Douro (Portugal)

Professor Universidade Estadual de Montes Claros, Grupo de Pesquisa em psicologia do sporte, exerc cio esa de ocupacional e m dia GIPESOM (Montes Claros - Brasil)

jclafeta@yahoo.com.br

Dur es, G. M., et al., (2015). Massagem na sa de laboral: Influ ncia no comportamento motor e estresse ocupacional. Educaci n F sica y Deporte, 34 (2), 555-574 Jul.-Dic. http://doi.org/10.17533/udea.efyd.v34n2a11 


\section{MARIA DE FATIMA MATOS MAIA}

Doutora pela Universidade Tras-os-Montes e Alto Universidade Tras-os-Montes e Alto Douro(Portugal).

Professora Universidade Estadual de Montes Claros, Grupo de Pesquisa em psicologia do sporte, exerc cio esa de ocupacional e m dia GIPESOM (Montes Claros - Brasil). mfatimaia@yahoo.com.br

\section{MARCEL GUIMARÃES DA SILVEIRA}

Mestre pela Universidade Tras-os-Montes e Alto Douro (Portugal)

Professor Universidade Estadual de Montes Claros, Grupo de Pesquisa em psicologia do sporte, exerc cio esa de ocupacional e $m$ dia GIPESOM (Montes Claros - Brasil)

Marcel.silvera3@gmail.com

\section{JEANE MENDES GONÇALVES}

Graduada (Bacherel) em Educaçao F sica pela Universidade Estadual de Montes Claros (Brasil).

jeane12@ig.com.br 


\section{RESUMO}

Objetivo: verificar os benef cios da massagem laboral no estresse ocupacional e comportamento motor. $M$ todo: a amostra foi composta por $31 \mathrm{t}$ cnicos administrativos, subdivididos de forma aleat ria em um grupo experimental e outro controle, que foram submetidos no pr e p s-teste ao exame da flexibilidade, força de preens o manual e aos question rios de estresse ocupacional. No tratamento dos dados recorreu-se estat stica inferencial, aos testes Kolmogorov Smirnov, Shapiro-Wilk, Wilcoxon, $t$ de Student para amostras pareadas, teste $t$ de Student para amostras independentes e Mann-Whitney, com n vel de signific ncia de 5\%. Resultado: nos efeitos agudos verificou-se que o grupo experimental apresentou uma melhora significativa nos $\mathrm{n}$ veis de força dos membros superiores direito $(p=0.039)$, esquerdo $(p=0.026)$, flexibilidade lateral da cabeça direita $(p=$ $0.039)$, apley direito $(p=0.011)$ e apley esquerdo $(p=0.010)$. Na an lise dos efeitos $\mathrm{cr}$ nicos, $\mathrm{n}$ o foram visualizados diferenças significativas no grupo experimental, embora o grupo controle apresentasse uma reduç o significativa da força de preens o direita $(p=0.001)$, esquerda $(p=0.017) \mathrm{e}$ cadeia posterior $(p=0.035)$.

PALAVRAS CHAVE: Massagem laboral, Sa de ocupacional, Comportamento motor, Estresse ocupacional.

\section{RESUMEN}

Objetivo: comprobar los beneficios del masaje de trabajo en el estr s laboral y el comportamiento motor (flexibilidad del cuerpo y fuerza de presi $n$ ) del personal administrativo. $M$ todo: la muestra fue compuesta por $31 \mathrm{t}$ cnicos administrativos, fraccionados en pre y pos test al examen de la flexibilidad, fuerza de la prensi $n$ manual y de los cuestionarios de estr $\mathrm{s}$ laboral. En el tratamiento de los datos se recurri a la estad stica inferencial, a los test Kolmogorov Smirnov, Shapiro-Wilk, Wilcoxon, $t$ de Student para las muestras pareadas, test $t$ Student para las muestras independientes y Mann-Whitney, con nivel de significaci n de 5\%. Resultado: en los efectos agudos se encontr que el grupo experimental mostr una mejor a significativa en los niveles de la fuerza de los miembros superiores derecho $(p=0.039)$, izquierdo $(p=0.026)$, flexibilidad lateral de la cabeza a la derecha $(p=0.039)$, apley derecho $(p=0.011)$ y apley izquierdo $(p=0.010)$. En el an lisis de los efectos $\mathrm{cr}$ nicos, no hubo diferencias significativas en el grupo experimental, mientras que el grupo control tuvo una reducci $\mathrm{n}$ significativa en la fuerza de prensi $\mathrm{n}$ derecha $(p=0.001)$, izquierda $(p=0.017)$ y cadena posterior $(p=0.035)$.

PALABRAS CLAVE: Masaje en el trabajo, Salud ocupacional, Comportamiento motor, Estr s en el trabajo. 


\section{ABSTRACT}

Aim: to verify the benefits of labor massage in occupational stress and motor behavior. Method: the sample consisted of 31 administrative staff, divided randomly into an experimental group and a control, who underwent preand post-test to the examination of flexibility, to handgrip strength and to the occupational stress questionnaire. In data processing, it appealed to the inferential statistics and to the testes: Kolmogorov Smirnov, Shapiro-Wilk, Wilcoxon, Student t test for paired samples, Student t test for independent samples and the Mann-Whitney test, with significance level of 5\%. Results: in acute effects it was found that the experimental group showed a significant improvement in the strength levels of the right $(p=0.039)$ and left $(p=0.026)$ upper limbs; lateral flexibility of the head to the right $(p=0.039)$, right apley $(p=0.011)$ and left apley $(p=0.010)$. In the analysis of chronic effects, significant differences were not displayed in the experimental group, while the control group had a significant reduction in right grip $(p=0.001)$ and left $(p=0.017)$ strengths and posterior chain $(p=0.035)$.

KEYWORDS: Labor massage, Occupational health, Motor behavior, Occupational stress. 


\section{INTRODUÇÃO}

A industrializaç o acelerada, aliada ao imediatismo financeiro das empresas e a implantaç o de novas tecnologias, exp e constantemente o trabalhador a $v$ rios fatores de riscos dentro do ambiente laboral (Batiz et al., 2013; Dal Pai et al., 2014). Algumas profiss es est o mais predispostas aos problemas ocupacionais, devido ao ritmo de trabalho, a repetitividade, o excesso de tarefas e a falta de pausa durante a jornada. Diante deste quadro, as doenças ocupacionais acometem a sa de do trabaIhador, afetando as estruturas musculoesquel ticas, podendo provocar Dist rbios Osteomusculares Relacionados ao TrabaIho - DORTs (Hugue \& Pereira, 2011).

A profiss o de $t$ cnico administrativo, que ser abordada no presente estudo, um exemplo que se enquadra dentre as caracter sticas citadas acima, onde um conjunto de fatores alteram e mudam o ambiente laboral, trazendo novas exig ncias, novas agress es, gerando sobrecarga f sica e psicol gica e consequentemente patologias como DORTs e estresse, que podem vir a afastar o trabalhador de suas atividades.

$\mathrm{V}$ rias alternativas e caminhos $\mathrm{s}$ o sugeridos para aliviar a jornada do trabalhador, neste contexto surge a Ergonomia, que

uma rea do conhecimento, cuja ess ncia transformar o trabalho em seus diferentes aspectos, adaptando-o s caracter sticas e aos limites do trabalhador (Abrah o et al., 2011).

Desta maneira, diversas empresas $t \mathrm{~m}$ investido na organizaç o do trabalho, buscando alternativas, que tragam benef cios aos colaboradores, promovendo assim a qualidade de vida no ambiente laboral. Uma das alternativas ergon micas utilizadas s o os Programas de Gin stica Laboral (PGL), que s o intervenç es realizadas durante a jornada de trabalho.

Segundo Castro et al. (2011) a gin stica laboral (GL) um programa de exerc cios $f$ sicos realizado no ambiente laboral, durante o expediente voltado para os trabalhadores, pois as au- 
las s o montadas considerando-se as atividades desempenhadas durante a jornada, de modo a relaxar a musculatura mais exigida e exercitar as menos solicitadas.

As sess es s o compostas por alongamentos, exerc cios localizados e respirat rios, din micas recreacionais e massagens (Lima, 2003). As t cnicas de massagem podem ser realizadas em grupo ou individualmente. A pr tica dentro de um PGL tem se mostrado essencial, uma vez que tem contribu do sobremaneira para a ades o e aumento da procura dos funcion rios $\mathrm{s}$ sess es, tudo isso em virtude das melhoras acarretadas vida dos trabalhadores. A massagem, aliada aos componentes de uma sess o de GL, tem produzido in meros benef cios, dentre os quais pode-se citar: a diminuiç o da tens o, da fadiga, das dores e melhoria do humor, sendo utilizada como uma ferramenta preventiva (Lima, 2003; Moretti \& Lima 2010).

Entretanto, $\mathrm{n}$ o $\mathrm{h}$ dispon vel na literatura pesquisas que comprovem os benef cios de um programa de massagem laboral sa de ocupacional de $\mathrm{t}$ cnicos administrativos. Neste sentido, o presente estudo tem por objetivo verificar quais $\mathrm{s}$ o os benef cios da massagem laboral no comportamento motor (flexibilidade e força muscular) e n vel de estresse ocupacional.

\section{METODOLOGIA}

O presente estudo caracterizou-se como uma pesquisa quase experimental, uma vez que foi realizado pr -teste e p s-teste, com um grupo experimental e um grupo controle. A pesquisa quase experimental apresenta um delineamento "em que o experimentador busca maior correspond ncia em ambientes do mundo real, ao mesmo tempo em que controla o maior $\mathrm{n}$ mero poss vel de ameaças validade interna" (Thomas et al., 2012, p. 365).

Este trabalho respeitou as normas ticas estabelecidas pelo Termo de Consentimento do Comit de tica, garantindo o sigi- 
lo da identidade dos indiv duos envolvidos e foi aprovado pelo Comit de tica da Universidade Estadual de Montes Claros, com n mero do CAAE: 05608412.7.0000.5146.

A populaç o desta investigaç o foi constitu da por funcio$\mathrm{n}$ rios que exercem atividades laborais na funç o de $t$ cnico administrativo em uma instituiç o $\mathrm{p}$ blica de ensino superior do munic pio de Montes Claros, Minas Gerais - Brasil.

A amostra foi composta por 31 funcion rios, sendo selecionados de forma intencional e por conveni ncia, independentemente do g nero e da idade. De acordo com Barros \& Lehefeld (1986) os elementos da amostragem intencional s o escolhidos de forma que estejam relacionados dentro das caracter sticas estabelecidas nos crit rios de inclus o.

A amostra foi subdividida aleatoriamente em dois grupos, sendo: o grupo experimental - GE $(n=18)$ e o grupo controle GC $(n=13)$. O GE participou de 10 sess es da massagem laboral (ML) ministradas duas vezes por semana, durante cinco semanas e receberam orientaç es ergon micas (OE). O GC recebeu apenas orientaç es ergon micas (orientaç es posturais e do posicionamento correto do mobili rio).

Ap s a aprovaç o do Comit de tica foi realizado um estudo piloto, com o objetivo de calibrar os instrumentos que foram utilizados, al $\mathrm{m}$ de treinar os avaliadores para a aplicaç o dos testes e question rios, de modo a se tornaram aptos a participarem da coleta junto aos funcion rios.

Para a coleta de dados desta investigaç o foram utilizados os question rios Escala de Estresse Percebido - EEP (Luft et al., 2007) e estresse no trabalho (Paschoal \& Tamayo, 2004), uma trena antropom trica da marca Sanny, com precis o de um cm, dinam metro port til da marca Takei Physical Fitness Test, Cadeira de quick massagem Mex Massage.

No pr e p s-teste, tanto os participantes do grupo experimental como do controle, foram submetidos avaliaç o da flexibilidade (cintura escapular e cadeia posterior), força de preens o 
manual direita e esquerda, al $\mathrm{m}$ de responderem aos question rios da escala de estresse percebido e de estresse no trabalho.

Ap s a aplicaç o dos testes preliminares, os membros do grupo experimental foram submetidos massagem laboral no segmento cervical, tor cico e da cintura escapular, com t cnicas de efleurage (manobra de deslizamento em retorno venoso), alisamento (deslizamento em direç o centr fuga), petrissage (t cnicas de amassamento para relaxamento), liberaç o miofascial (manobra de alongamento das $f$ scias musculares), vibraç o (t cnica de oscilaç es vibrat rias manuais) e orientaç es posturais. J o grupo controle recebeu apenas as orientaç es posturais.

Para avaliar os efeitos agudos (força e flexibilidade) no GE foi realizado o pr -teste e logo ap s a primeira sess o de massagem foi aplicado o p s-teste. J para avaliar os efeitos $\mathrm{cr}$ nicos no GE foi realizado o pr -teste e ap s um dia, posterior dez sess es de massagem e orientaç es ergon micas, foi aplicado o p s-teste (flexibilidade, força, escala de estresse percebido e estresse no trabalho). No entanto, no GC s foram analisadas as respostas cr nicas, na qual foi aplicado o pr -teste, ministrado as orientaç es ergon micas e ap s cinco semanas foi realizado o p s-teste.

Os dados foram tratados estatisticamente atrav $s$ da an lise inferencial, da $\mathrm{m}$ dia e do desvio padr o, com o programa SPSS (vers o18). Todas as vari veis foram submetidas aos testes de Kolmogorov Smirnov e de Shapiro-Wilk. Para testar os efeitos do experimento no pr -teste e no $\mathrm{p}$ s-teste, recorreram-se aos testes $t$ de Student para amostras pareadas e de Wilcoxon. Para comparar os resultados encontrados entre os grupos, empregaram-se os testes $t$ de Student, para amostras independentes e de Mann-Whitney, com um n vel de signific ncia de 5\%.

\section{RESULTADOS}

A amostra desta investigaç o foi composta por $31 \mathrm{t}$ cnicos administrativos, que atuam numa instituiç o de ensino superior $p$ blico 
de Montes Claros (MG). Destes, 58,1\% ( $n=18)$ dos participantes eram do grupo experimental e $41,9 \%(n=13)$ participaram do grupo controle. A $m$ dia de idade destes sujeitos foi de $28.7( \pm 9,42)$ anos, sendo $71 \%(n=22)$ do g nero feminino e $29 \%(n=9)$ do g nero masculino. Durante a anamnese constatou-se que apenas $6,5 \%(n=2)$ dos funcion rios avaliados relataram ter dist rbios patol gicos $\mathrm{cr}$ nicos (hipertens o arterial e enxaqueca) e 93,5\% $(n=29)$ eram indiv duos aparentemente saud veis.

Na tabela 1 a seguir est caracterizada a comparaç o dos resultados entre o g nero masculino e feminino das vari veis de força, flexibilidade e estresse. Assim, verificou-se dados homo$\mathrm{g}$ neos entre $\mathrm{g}$ neros na flexibilidade dos diversos segmentos corp reos e nos $\mathrm{n}$ veis de estresse. No entanto houveram diferenças significativas na força do membro superior direito - MSD $(p=0,008)$ e do membro superior esquerdo - MSE $(p=0,002)$.

No que se refere ao ndice estresse percebido e no trabalho, os resultados demonstraram que os funcion rios est o sendo submetidos a um $\mathrm{n}$ vel de estresse considerado moderado, $\mathrm{n}$ o se diferenciando de forma significativa em relaç o ao g nero.

Na tabela 2 abaixo est o apresentados os resultados no pr e p s-teste do grupo experimental para as vari veis de força e flexibilidade. A partir desses dados, observou-se uma melhora significativa na força no MSD $(p=0,040)$, na força do MSE $(p=0,026)$, na flexibilidade lateral $\mathrm{D}(p=0,039)$ e no Apley $\mathrm{D}(p=0,011)$.

Portanto estes dados demonstraram que a massagem apresentou benef cios de forma imediata (agudo) para manutenç o da flexibilidade e força muscular destas estruturas anat micas analisadas.

A tabela 3 exp e os resultados encontrados no $\mathrm{pr}$ e $\mathrm{p} \mathrm{s}$ -teste $\mathrm{cr}$ nico dos $\mathrm{n}$ veis de estresse, força e flexibilidade corp rea do grupo experimental. Neste sentido, evidenciou-se que a massoterapia no ambiente laboral promoveu benef cios para o aprimoramento dos componentes motores e na reduç o do estresse no trabalho. No entanto, esses resultados embora significativos de forma imediata, $\mathrm{n}$ o repercutiram a longo prazo ( $\mathrm{cr}$ nico). 


\begin{tabular}{|c|c|c|c|c|c|c|}
\hline \multirow{2}{*}{ Variável } & \multirow{2}{*}{$n$} & Masculino & \multirow{2}{*}{$n$} & Feminino & \multirow{2}{*}{$t / z$} & \multirow{2}{*}{$\boldsymbol{p}$} \\
\hline & & Média/DP & & Média/DP & & \\
\hline $\begin{array}{l}\text { Estresse } \\
\text { Percebido }\end{array}$ & 9 & $24,67 \pm 4,80$ & 22 & $25,09 \pm 4,42$ & 0,237 & 0,814 \\
\hline $\begin{array}{l}\text { Estresse no } \\
\text { Trabalho }\end{array}$ & 9 & $26,67 \pm 3,67$ & 22 & $28,69 \pm 10,8$ & 0,773 & 0,446 \\
\hline Força de MSD & 9 & $33,04 \pm 13,0$ & 22 & $22,03 \pm 8,17$ & $-2,838$ & $0,008 *$ \\
\hline Força de MSE & 9 & $32,72 \pm 12,7$ & 22 & $19,92 \pm 7,61$ & $-3,470$ & $0,002 *$ \\
\hline $\begin{array}{l}\text { Flexibilidade } \\
\text { lateral D }\end{array}$ & 9 & $6,27 \pm 2,48$ & 22 & $5,64 \pm 2,39$ & $-1,30$ & 0,194 \\
\hline $\begin{array}{l}\text { Flexibilidade } \\
\text { lateral E }\end{array}$ & 9 & $6,05 \pm 2,33$ & 22 & $5,79 \pm 2,23$ & $-7,49$ & 0,454 \\
\hline Rotação D & 9 & $6,05 \pm 2,42$ & 22 & $5,50 \pm 1,94$ & 0,66 & 0,508 \\
\hline Rotação E & 9 & $6,33 \pm 2,72$ & 22 & $5,58 \pm 1,86$ & $-0,70$ & 0,480 \\
\hline Apley D & 9 & $0,11 \pm 3,97$ & 22 & $1,09 \pm 3,46$ & 0,68 & 0,492 \\
\hline Apley E & 9 & $-1,05 \pm 4,03$ & 22 & $-0,22 \pm 4,00$ & $-0,63$ & 0,526 \\
\hline $\begin{array}{l}\text { Cadeia } \\
\text { Posterior }\end{array}$ & 9 & $-3,61 \pm 6,11$ & 22 & $-1,52 \pm 7,36$ & $-1,02$ & 0,308 \\
\hline
\end{tabular}

$* \mathrm{p} \leq .05$

Tabela 1. Análise comparativa das variáveis entre os gêneros no pré-teste.

\begin{tabular}{l|c|c|c|c|c}
\hline \multirow{2}{*}{ Variável } & \multirow{n}{*}{} & Pré-teste & Pós-teste & \multirow{2}{*}{ z } & \multirow{2}{*}{} \\
\cline { 3 - 4 } & & Média/DP & Média/DP & & \\
\hline Força MSD & 18 & $21,7 \pm 7,83$ & $23,85 \pm 9,35$ & $-2,06$ & $0,039 *$ \\
\hline Força MSE & 18 & $20,81 \pm 8,15$ & $21,80 \pm 9,31$ & $-2,22$ & $0,026 *$ \\
\hline Flexibilidade lateral D & 18 & $6,62 \pm 2,83$ & $6,21 \pm 2,61$ & $-2,06$ & $0,039 *$ \\
\hline Flexibilidade E & 18 & $6,69 \pm 2,57$ & $6,50 \pm 2,28$ & $-1,64$ & 0,100 \\
\hline Rotação D & 18 & $6,38 \pm 2,39$ & $6,26 \pm 2,22$ & $-1,05$ & 0,292 \\
\hline
\end{tabular}




\begin{tabular}{l|c|c|c|c|c}
\hline \multirow{2}{*}{ Variável } & \multirow{n}{*}{} & Pré-teste & Pós-teste & \multirow{2}{*}{ z } & \multirow{2}{*}{$\boldsymbol{p}$} \\
\cline { 3 - 4 } & & Média/DP & Média/DP & & \\
\hline Rotação E & 18 & $6,47 \pm 2,56$ & $6,08 \pm 2,05$ & $-1,40$ & 0,160 \\
\hline Apley D & 18 & $1,9 \pm 3,04$ & $2,69 \pm-4,00$ & $-2,54$ & $0,011 *$ \\
\hline Apley E & 18 & $0,38 \pm 4,00$ & $-0,70 \pm 7,49$ & $-2,56$ & $0,010 *$ \\
\hline Cadeia Posterior & 18 & $-0,69 \pm 7,48$ & $-0,69 \pm 7,48$ & 0,00 & 1,000 \\
\hline
\end{tabular}

$* p \leq 0,05$

Tabela 2. Análise comparativa do pré e pós-teste agudo dos níveis de força e flexibilidade do GE

\begin{tabular}{l|c|c|c|c|c}
\hline \multirow{2}{*}{ Variável } & \multirow{2}{*}{$\boldsymbol{n}$} & Pré-teste & Pós-teste & \multirow{2}{*}{ / z } & \multirow{2}{*}{$\boldsymbol{p}$} \\
\cline { 3 - 5 } & & Média/DP & Média/DP & & \\
\hline Estresse Percebido & 18 & $23,07 \pm 7,35$ & $24,05 \pm 5,25$ & 0,416 & 0,683 \\
\hline Estresse no Trabalho & 18 & $31,72 \pm 8,98$ & $30,01 \pm 10,42$ & 0,856 & 0,404 \\
\hline Força MSD & 18 & $21,76 \pm 7,76$ & $22,88 \pm 8,06$ & $-0,370$ & 0,711 \\
\hline Força MS & 18 & $20,81 \pm 8,15$ & $20,98 \pm 8,13$ & $-0,436$ & 0,663 \\
\hline Flexibilidade lateral D & 18 & $6,61 \pm 2,84$ & $6,07 \pm 2,47$ & 0,597 & 0,550 \\
\hline Flexibilidade lateral E & 18 & $6,69 \pm 2,57$ & $5,88 \pm 2,57$ & $-1,170$ & 0,242 \\
\hline Rotação lateral D & 18 & $6,38 \pm 2,39$ & $6,17 \pm 2,81$ & $-0,285$ & 0,776 \\
\hline Rotação lateral E & 18 & $6,47 \pm 2,56$ & $5,78 \pm 3,08$ & 0,653 & 0,514 \\
\hline Apley D & 18 & $1,9 \pm 3,04$ & $2,19 \pm 3,58$ & 0,485 & 0,627 \\
\hline Apley E & 18 & $0,38 \pm 4,00$ & $-0,40 \pm 5,20$ & 0,035 & 0,972 \\
\hline Cadeia Posterior & 18 & $-0,69 \pm 7,48$ & $0,19 \pm 7,67$ & $-0,912$ & 0,362 \\
\hline
\end{tabular}

* $p \leq 0,05$

Tabela 3. Análise comparativa pré e pós-teste crônico dos níveis de estresse, força e flexibilidade do GE 
A tabela 4 apresenta a an lise comparativa do grupo controle no pr e p s-teste $\mathrm{cr}$ nico dos $\mathrm{n}$ veis de estresse, força e flexibilidade corporal. Os resultados demonstraram $n$ o haver mudanças significativas nas vari veis investigadas. Entretanto, ocorreu uma reduç o significativa na força de preens o manual direita $(p=0,001)$, na força do MSE $(p=0,017)$ e na flexibilidade da cadeia posterior $(p=0,035)$.

\begin{tabular}{|c|c|c|c|c|c|}
\hline \multirow{2}{*}{ Variável } & \multirow{2}{*}{$n$} & Pré-teste & Pós-Teste & \multirow{2}{*}{$t / z$} & \multirow{2}{*}{$\mathbf{p}$} \\
\hline & & Média/DP & Média/DP & & \\
\hline Estresse percebido & 13 & $25,46 \pm 4,37$ & $26,69 \pm 3,88$ & $-1,655$ & 0,124 \\
\hline Estresse no trabalho & 13 & $23,07 \pm 7,35$ & $22,84 \pm 7,36$ & 0,492 & 0,632 \\
\hline Força MSD & 13 & $29,98 \pm 12,72$ & $27,11 \pm 11,64$ & 4,622 & 0,001 * \\
\hline Força MSE & 13 & $27,56 \pm 13,19$ & $25,60 \pm 13,11$ & 2,772 & 0,017 * \\
\hline Flexibilidade lateral D & 13 & $4,72 \pm 0,84$ & $4,76 \pm 0,75$ & 0,725 & 0,482 \\
\hline Flexibilidade lateral E & 13 & $4,27 \pm 0,76$ & $4,82 \pm 0,72$ & $-0,854$ & 0,410 \\
\hline Rotação D & 13 & $4,65 \pm 0,82$ & $4,51 \pm 0,56$ & 1,017 & 0,329 \\
\hline Rotação E & 13 & $4,86 \pm 0,63$ & $4,75 \pm 0,61$ & 1,148 & 0,273 \\
\hline Apley D & 13 & $-0,72 \pm 3,82$ & $-0,42 \pm 3,48$ & $-1,422$ & 0,180 \\
\hline Apley E & 13 & $-1,65 \pm 3,72$ & $-2,07 \pm 3,24$ & 0,759 & 0,462 \\
\hline Cadeia Posterior & 13 & $-4,11 \pm 5,95$ & $-4,50 \pm 6,14$ & 2,378 & $0,035 *$ \\
\hline
\end{tabular}

$* p \leq 0,05$

Tabela 4. Análise do pré e pós-teste crônico dos níveis de estresse, força, flexibilidade do Grupo Controle - GC

A tabela 5 a seguir demonstra os resultados observados na an lise comparativa (efeitos $\mathrm{cr}$ nicos) entre os grupos, das vari veis: força, flexibilidade e estresse. Dessa forma, verificou-se que a maioria das vari veis $\mathrm{n}$ o apresentaram diferenças estatisticamente significativas entre os grupos investigados. Houve 
diferença significativa apenas na força de preens o do membro superior direito $(p=0,014)$ e esquerdo $(p=0,051)$, com predo$\mathrm{m}$ nio dos participantes da massagem. No entanto, grande parte destas vari veis obtiveram resultados mais positivos no grupo experimental, enquanto os participantes que $\mathrm{n}$ o receberam a massoterapia laboral apresentaram resultados mais negativos.

\begin{tabular}{l|c|c|c|c|c|c}
\hline \multirow{2}{*}{ Variável } & \multirow{2}{*}{$\boldsymbol{n}$} & $\begin{array}{c}\text { Grupo } \\
\text { Experimental }\end{array}$ & \multirow{n}{*}{$n$} & $\begin{array}{c}\text { Grupo } \\
\text { Controle }\end{array}$ & \multirow{2}{*}{$\boldsymbol{U}$} & \multirow{2}{*}{$\boldsymbol{p}$} \\
& & Média / DP & & Média / DP & & \\
\hline Estresse Percebido & 18 & $-0,56 \pm 5,67$ & 13 & $1,23 \pm 2,68$ & 143,00 & 0,312 \\
\hline Estresse no Trabalho & 18 & $-1,72 \pm 8,53$ & 13 & $-0,23 \pm 1,69$ & 151,00 & 0,183 \\
\hline Força de MSD & 18 & $1,13 \pm 6,95$ & 13 & $-2,87 \pm 2,24$ & 56,00 & $0,014 *$ \\
\hline Força de MSE & 18 & $0,17 \pm 3,92$ & 13 & $-1,97 \pm 2,55$ & 68,00 & $0,051 *$ \\
\hline Flexibilidade lateral D & 18 & $-0,54 \pm 3,50$ & 13 & $0,46 \pm 0,23$ & 138,00 & 0,417 \\
\hline Flexibilidade lateral E & 18 & $-0,81 \pm 3,44$ & 13 & $0,10 \pm 0,42$ & 150,50 & 0,183 \\
\hline Rotação D & 18 & $-0,21 \pm 3,85$ & 13 & $-0,14 \pm 0,49$ & 130,50 & 0,594 \\
\hline Rotação E & 18 & $-0,68 \pm 4,05$ & 13 & $-0,12 \pm 0,36$ & 129,50 & 0,622 \\
\hline Apley D & 18 & $0,28 \pm 1,99$ & 13 & $0,30 \pm 0,76$ & 121,50 & 0,859 \\
\hline Apley E & 18 & $0,01 \pm 4,19$ & 13 & $-0,42 \pm 2,01$ & 110,50 & 0,798 \\
\hline Cadeia Posterior & 18 & $0,50 \pm 9,59$ & 13 & $-0,38 \pm 0,58$ & 69,50 & 0,056 \\
\hline
\end{tabular}

$* p \leq 0,05$

Tabela 5. Análise comparativa dos efeitos crônicos da massoterapia no estresse, força e flexibilidade entre os grupos (Pós - Pré-teste)

\section{DISCUSSÃO}

A presença de dores e de outras alteraç es funcionais advindas dos Dist rbios Osteomusculares Relacionados ao Trabalho (DORT) pode desencadear sintomas depressivos e de ansieda- 
de, que muitas vezes s o acompanhados de ang stia em relaç o a um futuro incerto, fazendo com que o trabalhador perca sua identidade ficando inseguro no ambiente de trabalho, familiar e social (Pessoa et al., 2010; Martins, 2005).

Quanto a utilizaç o do corpo, Foucault (2006, p.80) salienta que "o corpo foi investido pol tica e socialmente como força de trabalho". Neste sentido o trabalhador seria moldado para produzir aos governos e entidades privadas o retorno financeiro atrav s da força de produç o o que acarreta um disp ndio pessoal de energia em suas funç es laborais de acordo com a estrutura corporal pretendida.

No decorrer da hist ria da humanidade, a massagem foi usada por distintas civilizaç es como um elemento para abrandar desconfortos, tratar e prevenir doenças, suavizar o cansaço, auxiliar um adequado per odo de sono e gerar o bem estar (Moretti \& Lima, 2010).

$\mathrm{Na}$ atualidade a massoterapia tem conquistado um grande espaço no desenvolvimento de estudos cient ficos e nos procedimentos adotados por diversos profissionais da sa de, sendo utilizada como modalidade profil tica e da reabilitaç o de diversos dist rbios osteomusculares e cinesiofuncionais.

Para efeito de conceituaç o e entendimento do estudo, Conceiç o \& Dias (2004) estabelecem que o efeito agudo ou imediato est relacionado a toda manifestaç o fisiol gica que acontece durante e imediatamente ap s a pr tica de alguma atividade $\mathrm{f}$ sica. $\mathrm{J}$ o efeito $\mathrm{cr}$ nico, relaciona-se com um per odo longo ao decorrer do tempo, em que o estresse provoca um efeito adaptativo, sendo que tais efeitos podem permanecer por determinado tempo ap s suspender a atividade.

Neste sentido os resultados encontrados nesse estudo $\mathrm{v} \mathrm{m}$ ao encontro da literatura, pois Nordschow \& Bierman (1962), desenvolveram um estudo para avaliar os efeitos da massagem laboral na flexibilidade posterior do tronco em 25 pessoas. $\mathrm{Na}$ coleta de dados foram analisadas a flexibilidade segmentar de 
cada participante antes e depois da massagem com duraç o de 30 minutos, sendo realizada na coluna vertebral e membros inferiores. Todos os pacientes do estudo demonstraram ganho de flexibilidade do tronco, ap s serem submetidos massagem, comparados leitura pr e p s-teste. Concluiu-se que a massagem de forma imediata pode criar um relaxamento nos $\mathrm{m}$ sculos volunt rios, embora os mecanismos $\mathrm{n}$ o fossem elucidados.

Martins (2005), ap s realizar quatro meses de sess es de gin stica laboral (que continha massagem nos seus procedimentos), duas vezes por semana junto a 26 funcion rios da Universidade Federal de Santa Catarina, analisou a força e flexibilidade ap s programa de exerc cios e observou que houve uma melhoria nessas vari veis do comportamento motor.

Sisko et al., (2011) desenvolveram um estudo para avaliar os efeitos de um programa de massagem na amplitude articular de movimento (ADM) e dor musculoesquel tica em 19 funcio$\mathrm{n}$ rias de um setor administrativo. Para isso, foram submetidas a sess es de quick massage duas vezes por semana durante um $\mathrm{m}$ s. Ap s pr /p s-teste com an lise angular e desconforto musculoesquel tico de Cornell, observou-se aumento significativo $(p<0,05)$ da ADM da flex o lateral e extens o cervical e reduç o significativa da dor no pescoço e parte superior das costas. Contudo, constataram respostas bem mais efetivas e duradouras ap s um $\mathrm{m}$ s de intervenç o, em comparaç o ao efeito agudo (em apenas uma sess o).

Dur es et al. (2011) investigaram os benef cios da massagem laboral na flexibilidade em auxiliares de limpeza. Participaram deste estudo 20 trabalhadores de uma instituiç o de ensino superior, subdivididos em um grupo experimental e controle. Os resultados verificaram $n$ veis reduzidos de flexibilidade em $\checkmark$ rios segmentos corp reos. A massagem promoveu um incremento na flexibilidade do grupo experimental, uma vez que ao serem avaliados os ndices obtidos entre os grupos, averiguaram-se diferenças significativas na flexibilidade da rotaç o cervi- 
cal esquerda $(p=0,013)$, flex o lateral esquerda $(p=0,001)$ e direita $(p=0,015)$, bem como da cintura escapular $(p=0,018)$.

No presente estudo a massagem $\mathrm{n}$ o promoveu de forma $\mathrm{cr}$ nica um aumento significativo na flexibilidade dos diversos segmentos avaliados, sendo que os resultados foram leves e discretos. Esses resultados podem ter sido influenciados pela duraç o e frequ ncia das sess es de massoterapia.

$O$ estresse um dos males que mais prejudica a qualidade de vida dos trabalhadores, levando a uma reduç o do rendimento laboral e elevaç o da tens o muscular (Konopatzki et al., 2001). Este mecanismo agressor a sa de fsica e mental pode ser explicada pelo crescente $n$ vel de cobrança estabelecido pelas empresas que, com intuito de aumentarem a produtividade e o lucro, acabam por sobrecarregarem os trabalhadores, gerando dessa forma, altos $\mathrm{n}$ veis de estresse.

Martins (2005) verificou os $n$ veis de estresse em trabalhadores da reitoria da Universidade Federal de Santa Catarina. Como resultados o autor evidenciou $n$ veis elevados de estresse naquele ambiente laboral. Por outro lado, os resultados obtidos no presente estudo demonstram que estes trabalhadores apresentaram ndices moderados de estresse ocupacional.

Com os resultados dessa investigaç o demonstrou-se uma reduç o no ndice de estresse no trabalho com a aplicaç o da massoterapia, embora de forma $n$ o significativa. Por outro lado, tanto o GE como o GC, apresentaram um aumento dos $n$ veis de estresse percebidos, que podem ter sido influenciados por fatores extra-ocupacionais (pessoais).

Contrastando esses estudos, Hodge et al. (2002) analisaram o potencial da massagem como $t$ cnica eficaz para a reduç o do estresse no ambiente de trabalho. A amostra foi composta por 100 volunt rios de ambos os g neros com idade de 25 a 60 anos, que desenvolviam atividades laborais em um hospital universit rio.

A amostra foi subdividida em dois grupos, sendo que o GE recebeu uma massagem com press o de suave a $m$ dia inten- 
sidade, na parte superior do tronco por 20 minutos, duas vezes por semana, durante duas semanas e os sujeitos do GC permaneciam em uma sala para descansar pelo mesmo per odo de duraç o. Os resultados indicaram que, em comparaç o com o grupo controle, os indiv duos que receberam a massagem mostravam melhor controle emocional (Hodge et al., 2002).

Lafet et al. (2012) realizaram uma revis o bibliogr fica entre 2000 a 2012, sobre os efeitos da massagem laboral nas algias do trabalho. A literatura preconiza que a massagem no ambiente de trabalho contribui para reduzir os dist rbios Igicos ocupacionais, sobretudo associados aos desequil brios neuromusculares, estresse, fadiga ocupacional, posturas inadequadas e sobrecarga $f$ sica excessiva.

Conforme Moretti \& Lima (2010) o principal efeito da massagem proveniente de uma via reflexa (sedaç o neuromuscular), que diminui a estimulaç o dos neuroceptores (terminaç es nervosas livres), que frequentemente indicada pra reduzir a dor, a ansiedade e amenizar o estresse e os dist rbios Igicos ocupacionais.

Borges et al., (2014) no estudo com trabalhadores de enfermagem da cidade de Carapicu ba/SP, conclu ram que a massagem mostrou-se uma terapia complementar eficaz para diminuiç o de lombalgia ocupacional em trabalhadores de enfermagem, medida que melhorou os escores de dor, alterando-os de dor moderada para leve. Nesta investigaç o a massagem obteve um resultado expressivo $(d=4,59)$, correspondente a $86 \%$ de reduç o dos $n$ veis de dor.

Assim, pode-se inferir que a massagem realizada no ambiente de trabalho constitui uma medida preventiva que auxilia no controle da sobrecarga $f$ sica, principalmente associada aos comportamentos motores e do estresse ocupacional. No entanto, para maior compreens o dos seus benef cios para os trabalhadores, sugerem-se novas investigaç es que envolvam diferentes profiss es e diversas $t$ cnicas massoter picas, duraç $\mathrm{O}$ e frequ ncia de aplicaç o. 


\section{CONCLUSÃO}

Este estudo apresentou dados referentes aos benef cios (efeitos agudos/cr nicos) da massagem laboral no comportamento motor (força e flexibilidade) e $\mathrm{n}$ veis de estresse ocupacional em servidores $t$ cnico-administrativos.

Com relaç o s respostas imediatas (agudo) da massagem laboral no grupo experimental, constatou-se uma melhora significativa nos $n$ veis de força dos membros superiores e flexibilidade segmentar da cabeça e ombros.

No que se refere aos efeitos $\mathrm{cr}$ nicos, $\mathrm{n}$ o foram evidenciadas mudanças significativas no estresse, flexibilidade e força do grupo experimental. J no grupo controle, ocorreu uma reduç o significativa da força e da flexibilidade da coluna vertebral (cadeia posterior). O estresse, a flexibilidade dos ombros e da cabeça $\mathrm{n}$ o apresentaram mudanças significativas.

Ao comparar os resultados encontrados entre os grupos, observou-se que grande parte das vari veis $\mathrm{n}$ o apresentaram diferenças estatisticamente significativas. Por $\mathrm{m}$, houve diferença significativa apenas na força de preens o dos membros superiores, com predom nio dos participantes da massagem.

Diante do exposto, conclui-se que a massagem no ambiente laboral constitui uma importante ferramenta para o aprimoramento do comportamento motor, promovendo um aumento da força e da flexibilidade. Em relaç o ao estresse ocupacional, esta medida preventiva exerce uma discreta influ ncia para o seu controle.

\section{REFERÊNCIAS}

1. Abrah o, J., Sznelwar, L., Silvino, A., Sarmet, M., \& Pinho, D. (2011). Introduç o ergonomia: da pr tica teoria. S o Paulo: Blucher.

2. Barros, A. J. P., \& Lehefeld, N. A. S. (1986). Fundamentos de metodologia: um guia para inicializaç o cient fica. S o Paulo: Mc Graw-Hill. 
3. Batiz, E. C., Nunes, J. I. S., \& Licea, O. E. A. (2013). Preval ncia dos sintomas musculoesquel ticos em movimentadores de mercadorias com carga. Produç o, 23(1), 168-177.

4. Borges, T. P., Kurebayashi, L. F. S., \& Silva, M. J. P. (2014). Lombalgia ocupacional em trabalhadores de enfermagem: massagem versus dor. Revista da Escola de Enfermag da USP, 48(4):669-75.

5. Castro, E., M nera, J. E., Sanmart n, M., Valencia, N. A., Valencia, N. D., \& Gonz lez, E. V. (2011). Efectos de un programa de pausas activas sobre la percepci $\mathrm{n}$ de des rdenes $\mathrm{m}$ sculo-esquel ticos en trabajadores de la Universidad de Antioquia. Educaci n F sica y Deporte, 30(1), 389-399.

6. Conceiç O, A. O, \& Dias, G. A. S. (2004). Alongamento Muscular: uma vers o atualizada. Revista Lato \& Sensu, 5(1), 136-141.

7. Dal Pai, D., Lautert, L., Tavares, J. P., Souza Filho, G. A., Dornelles, A. N., \& Merlo, A. R. C. (2014). Repercuss es da aceleraç o dos ritmos de trabalho na sa de dos servidores de um juizado especial. Sa de e Sociedade, 23(3), 942-952.

8. Dur es, G. M., Ferreira, M. A. M., Silveira, M. G., \& Lafet , J. C. (2011). Efeitos da massagem laboral na flexibilidade articular e na reduç o do stress ocupacional em auxiliares de limpeza. Revista Norte-Mineira de Educaç o F sica, 1(1), 34-51.

9. Foucault, M. (2006). O nascimento da medicina social. In M. Foucault, Microf sica do poder (22a ed.) (pp. 79-98). Rio de Janeiro: Graal.

10. Hodge, M., Robinson, C., Boehmer, J., Klein, S., \& Ullrich, S. (2002). Employee outcomes following work - site acupressure and massage. In G. J. Riched, Massage therapy: the evidence for practice (pp.191202). St.Louis: Mosby.

11. Hollis, M. (1993). Practical exercise therapy ( $3^{\text {rd }}$ ed.). USA: Blackwell Science Ltda, Oxford.

12. Hugue, T. D., \& Pereira J nior, A. A. (2011). Preval ncia de dor osteomuscular entre os servidores administrativos da Unifebe. Revista da Unifebe, 9, 1-9.

13. Konopatzki, A. C., Campos, A. T., \& Martins, C. (2001). Massoterapia na prevenç o das complicaç es do estresse. Fisioterapia em Movimento, 14(1), 35-43.

14. Lafet , J. C., Dur es, G. M, Gomes., F. M., Gonçalves, J. M., \& Silveira, M. G. (2012). Massagem laboral aplicada aos dist rbios Igicos ocupacionais: uma breve revis o. Revista Mineira de Educaç o F sica, 8, 541-552.

15. Lima, V. (2003). Gin stica laboral: atividade $f$ sica no ambiente de trabalho. S o Paulo: Phorte.

16. Luft, C. B., Sanches, S. O., Mazo, G. Z., \& Andrade, A. (2007). Vers o brasileira da Escala de Estresse Percebido: traduç o e validaç o para idoso. Revista de Sa de $P$ blica, 41(4), 606-615. 
17. Martins, C.O. (2005). Repercuss o de um programa de gin stica laboral na qualidade de vida trabalhadores de escrit rio. Santa Catarina, Brasil: Universidade Federal de Santa Catarina, Programa de P s-Graduaç o em Engenharia de Produç o da, Doutorado em Engenharia de Produç o.

18. Moretti, A., \& Lima, V. (2010). Massagem no ambiente de trabalho. S o Paulo: Phorte.

19. Nordschow, M., \& Bierman, W. (1962). Influence of manual massage on muscle relaxation: effect on trunk flexion. Journal of American Physical Therapy Association, 42(10), 653-657.

20. Paschoal, T., \& Tamayo, A. (2004). Validaç o da escala de estresse no trabalho. Estudos de Psicologia, 9(1), 45-52.

21. Pessoa, J. C. S., Cardia, M. C. G., \& Santos, M. L. C. (2010). An lise das limitaç es, estrat gias e perspectivas dos trabalhadores com LER/ Dort, participantes do grupo Profit-LER: um estudo de caso. Ci ncia \& Sa de Coletiva, 15(3), 821-830.

22. Si ko, P.K., Videm ek, M., \& Karpljuk, D. (2011). The effect of a corporate chair massage program on musculoskeletal discomfort and joint range of motion in office workers. Journal of Alternative and Complementary Medicine, 17(7), 617-22.

23. Thomas, J. R., Nelson, J. K., \& Silverman, S. J. (2012). M todos de pesquisa em atividade $f$ sica. ( $6^{\mathrm{a}}$ ed.). Porto Alegre: Artmed.

Recepción 10-06-2015

Aprobación: 02-11-2015 